\title{
INVESTIGATION OF THE EFFECTS OF FOOD PROCESSING AND MATRIX COMPONENTS ON THE ANALYTICAL RESULTS OF ELISA USING AN INCURRED GLIADIN REFERENCE MATERIAL CANDIDATE
}

\author{
K. TÖRÖK*, L. Hajas, Zs. Bugyi, G. Balázs and S. TÖMÖSKÖZI \\ Department of Applied Biotechnology and Food Science, Budapest University of Technology and Economics, \\ H-1111 Budapest, Szent Gellért tér 4. Hungary
}

(Received: 31 July 2013; accepted: 31 January 2014)

\begin{abstract}
Disorders induced by cereal proteins (e.g. wheat allergy, celiac disease) are widespread in human population. Since their only effective treatment is the avoidance of the problematic proteins, patients have to be familiar with the composition of food products. For checking special foods produced for them, proper analytical methods are necessary. At the moment, in gluten analysis there are no reference methods and reference materials which model real food matrices. During the production and experimental utilisation of our previously developed reference material candidate, numerous questions emerged. As our model product is a real food matrix, interactions can be present between gluten proteins and other macro and micro components. Fat content of the baked cookies is almost $20 \%$, which might affect the results of ELISA measurements. The detectable gluten content is significantly increasing after the defatting procedure, as a pre-treatment of samples. Moreover, baking is a common food processing step that might modify the structure of gluten proteins leading to denaturation and aggregation. In the soluble protein fraction the amount of low molecular weight proteins increases, while that of high molecular weight proteins decreases during the baking procedure.
\end{abstract}

Keywords: gluten determination, ELISA method, reference material, sample pre-treatment, protein denaturation

Wheat and other cereals are staples in human nutrition. Consumption of wheat-based food products does not cause any health problems for the majority of consumers but in some of them proteins of wheat and other cereals can cause adverse reactions. These hypersensitivity reactions mostly manifest as wheat allergy or celiac disease.

Wheat allergy is an IgE-mediated food disorder where the triggering proteins are gliadins, glutenins, and some albumin and globulin proteins. Celiac disease is an autoimmune entheropathy triggered by the ingestion of gluten and the related alcohol soluble fractions of rye and barley (Taylor \& Hefle, 2001; Briani et al., 2008; Cabera-Chavez \& Calderon de LA BARCA, 2010).

For detection and quantification of these proteins, application of reliable, validated analytical methods is required (BOye \& Godefroy, 2010). There are several analytical methods for detecting the target proteins or the presence of wheat and other cereals in foods, such as ELISA, immunoblotting, PCR and MS techniques. Currently the most commonly used method in routine analysis is ELISA (Koppelman \& Hefle, 2006).

* To whom correspondence should be addressed.
Phone: +36-1-463-3865; fax: +36-1-463-3855; e-mail: ktorok@mail.bme.hu

0139-3006/\$20.00 C 2015 Akadémiai Kiadó, Budapest 
The commercially available ELISA tests are using various antibodies for the specific detection of the target molecules. Furthermore, the sample preparation procedures may be different in the test kits. The accuracy of the methods and the comparability of the results are also questionable, since different standard materials or solutions are used for the calibration of the test kits. A reference method does not exist and there is a continuous debate on the type and utilisation of reference materials. Currently there is no available reference material that models a real, processed food matrix (Koppelman \& Hefle, 2006). In addition, storage proteins of wheat may be altered during food processing. The denaturation, structure changes, and complex formation can modify the solubility properties and the immunochemical reactivity of proteins (SATHe et al., 2005; GomaA \& Boye, 2013). Protein denaturation and the structure modification processes during heat treatment were studied mainly in baking trials (Singh \& MacRitchie, 2004) and insufficient information is available concerning the immuno-analytical reactions in simplified model systems (RUMBo et al., 2001). High temperature is leading to a decrease of extractable protein amount. In the soluble fraction, the amount of gliadin proteins increases, while the amount of glutenin proteins decreases. In spite of these facts, in most cases test kits do not offer a different extraction procedure for processed samples. Moreover, no other sample pre-treatment instructions are available for fatty or other type of complex food matrices.

To investigate and eliminate these problems, processed food matrices with defined gliadin content were developed on labscale by our research group (BugYI et al., 2012). In order to use these model products as reference materials, a number of requirements must be fulfilled. These are the defined gliadin concentration, homogeneous distribution of proteins, reproducible production procedure, and the definition of dedicated parameters, such as stability and storage properties (ISO, 1992; Koppelman \& Hefle, 2006).

To meet these requirements, several tests were performed with our model matrices, in which the recovery and homogeneity of gliadin were investigated and found to be satisfactory. Furthermore, different commercially available ELISA assays were used in these experiments and significant differences were observed among the results of ELISA methods. These discrepant results can be caused by the various sample preparation protocols, target molecules, standard materials, and alteration in the calculation of results. Besides, the gluten content was determined in all phases of the production, such as the powder mixture, the raw dough, and the final baked cookies were also investigated in order to determine the effects of food processing. Results of ELISA measurements showed a decrease of measurable gliadin protein content throughout the process, mainly in cookies compared to the raw dough. The background may be the changes in antibody binding activity and solubility of proteins (BUGYI et al., 2013).

In order to reveal the background of the phenomena mentioned above, the effects of two factors were examined in our current study. The first factor was defatting as a sample pretreatment step for ELISA measurements. After the defatting procedure, the changes in gluten solubility and in detectable protein amount were investigated. Moreover, the solubility properties and protein profile of wheat proteins were studied with SE-HPLC method. According to the literature, acetonitrile is the most suitable extraction solution for SE-HPLC measurements (SINGH, 2005; MANU \& RAO, 2008). ELISA methods are applying alcoholcontaining extraction solution for protein solubilisation. Therefore, the protein profiles obtained with SE-HPLC were also studied using an ethanol-containing extraction solvent and these data were compared to those obtained by using acetonitrile. 


\section{Materials and methods}

\subsection{Sample production}

The gliadin reference material candidate model products (baked cookies) were produced according to BUGYI and co-workers (2012). For the investigation of the effects of matrix components with ELISA methods these materials were applied. Theoretical concentration of the baked cookies was $10 \mathrm{ppm}$ gliadin (CV: 7.9\%).

For studying the processing steps with HPLC method, two modifications were realised in the process. The protein content of the new model products made for the SE-HPLC experiment was set to $2 \%$ to obtain a sufficiently high gliadin concentration for chromatographic measurements. Besides, in order to have a more realistic matrix and for the possibility to examine the whole protein profile of wheat, the previously applied PWG gliadin was replaced by wheat flour (variety MV Magvas, Martonvásár, Hungary) as wheat protein source. Homogeneity testing yielded coefficient of variation (CV) values below 10\%. For studying the effects of matrix components and the effects of production procedure, samples were taken from all phases of the production, i.e. from the wheat flour, the powder mixture of raw materials, the raw dough, and the baked cookies.

All samples were ground (Retsch AS 200, Basic), placed in closed sample containers, and stored at room temperature in the dark before analysis.

\subsection{Investigation of the effects of lipid extraction}

Fat content of our samples were determined with standard Soxtec extraction (SOXTEC SYSTEM HT, 1043 Extraction Unit, Tecator AB, Sweden, A.O.A.C. (2006), Method 2003.06).

The same Soxtec extractor was used for defatting the samples. In order to protect the proteins from further heat treatment, the samples were only rinsed by the condensed solvent (n-hexane 96\% a.r., Molar Chemicals) for $2 \mathrm{~h}$. After the extraction procedure, defatted samples were dried for $24 \mathrm{~h}$ at room temperature.

Two ELISA methods (R-Biopharm RIDASCREEN Gliadin Assay, R7001 and Romerlabs AgraQuant Gluten Assay, COKAL0248) were applied in our study. The manufacturer's instructions were followed for sample preparation (except for the defatting procedure) and for the implementation of the tests. For ELISA measurements three replicates were analyzed for each sample.

\subsection{Characterisation of protein profiles}

SE-HPLC method was used according to LARROQUE and BÉKÉs (2000), only the sample preparation was partly modified. The first extraction solution contained $50 \%(\mathrm{v} / \mathrm{v})$ acetonitrile $(\mathrm{ACN})$ and $1 \%(\mathrm{v} / \mathrm{v})$ triflouroacetic acid (TFA) similarly to the cited method, while $70 \%(\mathrm{v} / \mathrm{v})$ ethanol was also used as an alternative solvent. In both cases, $15 \mathrm{mg}$ of ground sample was extracted in $1 \mathrm{ml}$ extraction solution. The suspensions were shaken for $30 \mathrm{~min}(800 \times \mathrm{g})$ and centrifuged for $10 \mathrm{~min}(7000 \times \mathrm{g})$. The supernatant contained the soluble protein fractions (S1). The precipitate was dissolved in $1 \mathrm{ml}$ of the same extraction solution, then the suspension was sonicated for $40 \mathrm{sec}$ with the amplitude of $90 \%$ (UP 200H Ultrasonic Processor, Hielscher Ultrasonic GmbH, Germany). Next step was the centrifugation of the samples on 7000 $\times g$ for $10 \mathrm{~min}$. This second supernatant (S2) contained the insoluble protein fractions. The SE- 
HPLC measurements were carried out with Perkin Elmer Series 200 HPLC instrument and UV-VIS Diode Array Detector. The supernatants were separated on a Phenomenex Biosepsec-S4000 column at $25^{\circ} \mathrm{C}$. The flow rate was $1 \mathrm{ml} \mathrm{min}^{-1}$.

\subsection{Data analysis}

The analytical results of ELISA measurements were calculated using BioRad Microplate Manager 6 software. To analyse the results of SE-HPLC method TotalChrom Navigator software (version 6.2.1) was used. The determined protein fractions are demonstrated in Fig. 1 . The data were evaluated by the investigation of means and standard deviations. The calculated concentrations were compared by two-sample $t$-tests.

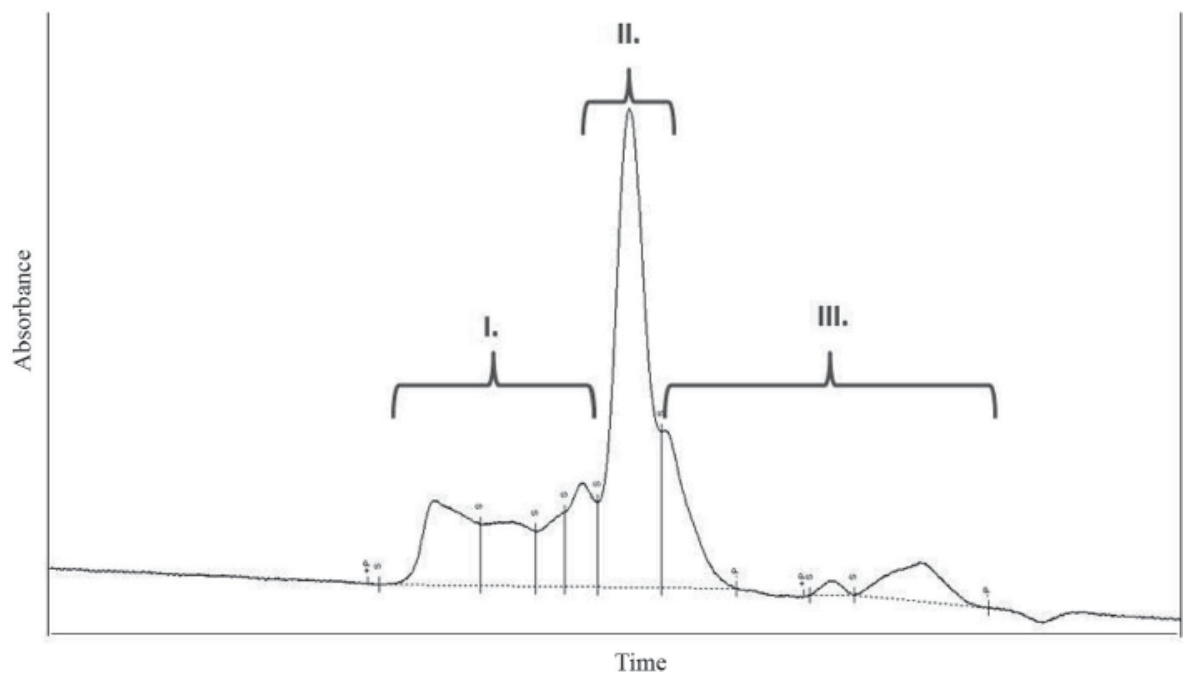

Fig. 1. Evaluation of SE-HPLC chromatogram. In case of native wheat flour: I: glutenins and $\omega$-gliadins, II: $\alpha-, \beta$-, $\gamma$-gliadins; III: low molecular weight proteins

\section{Results and discussion}

\subsection{Effects of defatting}

ELISA measurements of defatted and non-defatted cookies show significant differences in measurable gliadin concentration after lipid extraction (Fig. 2). The background of this phenomenon is not clear at the moment. As lipid extraction was successful, lipid-protein complexation in great extent is unlikely. Lipid content might affect gluten solubility and more importantly their affinity to the antibodies, though.

Instructions enclosed to commercially available ELISA kits do not prescribe defatting protocols for sample preparation of food samples. However, our results pointed out the importance of this question: a more complex sample preparation can improve the reliability of the results. With the help of our reference material candidates the rate of uncertainty originated from fat content or defatting pretreatment can also be determined. More experiments are planned to clarify the background and to work out the standard procedure of sample pre-treatment. 


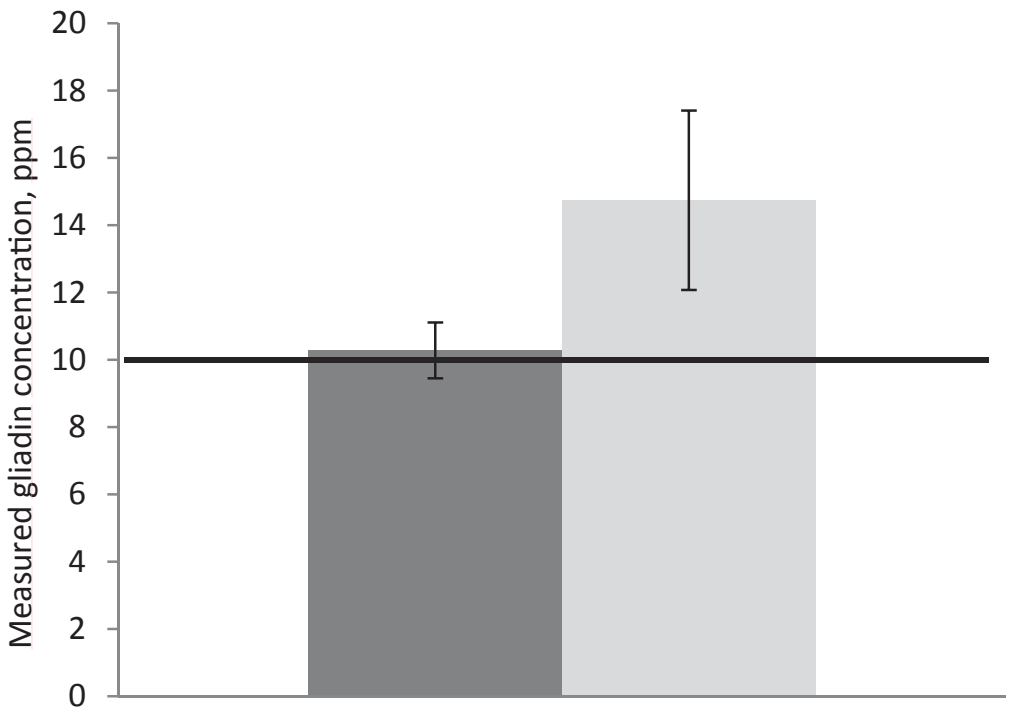

Fig. 2. Gliadin concentration determined by R-Biopharm RIDASCREEN Gliadin Assay in baked cookies. Concentrations are represented on dry matter basis. $\square$ : untreated cookie; $\square$ : defatted cookie

\subsection{Investigation on the background of decreasing measurable gluten concentration during processing}

The results of ELISA measurements show decreasing measurable gliadin content in cookies compared to the raw dough (BuGYI et al., 2012). This effect was observed with all ELISA kits we used but the extent of these discrepancies was different (BugYi et al., 2013). To explore the background of these phenomena the physical and chemical behaviour of wheat proteins had to be examined.

As the first step of our study we investigated and compared the size distribution profiles of proteins in four phases of the RM production: in the wheat flour, which was applied as protein source, in the powder mixture of raw materials, in the raw, freeze-dried dough, and in the baked cookies.

Using ACN, changes in characteristics were observed in protein profile throughout the production process (Fig. 3). The first phenomenon was a decrease in extractable protein content after baking by the decrease in the total area of the SE-HPLC chromatogram. Peak area of high molecular weight proteins decreased in the heat treated cookies. In contrast, the amount of low molecular weight proteins increased during the processing steps. Besides, profile of gliadin peaks did not change significantly. Chromatograms of insoluble fractions (Fig. 4) also show changes during food processing. Peak area of glutenins did not change, while the rate of gliadins and low molecular weight proteins are continuously increasing from wheat flour to baked cookies. 

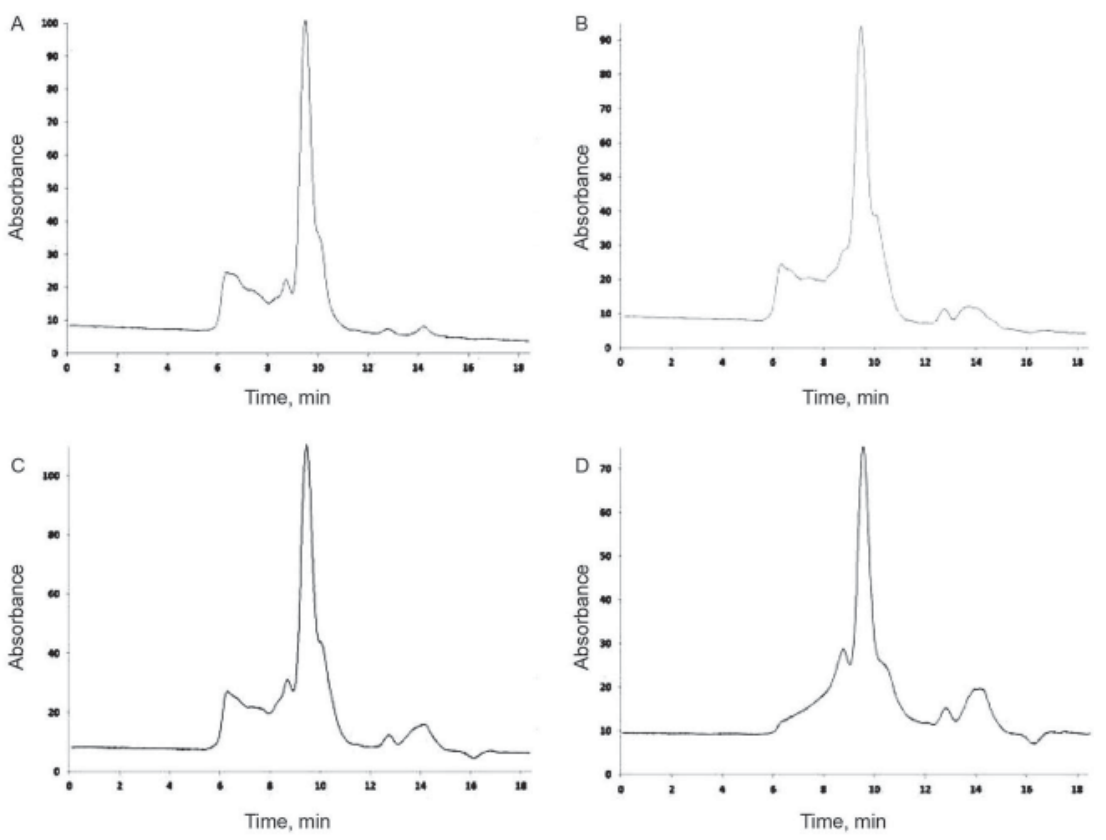

Fig. 3. Soluble fractions of A) wheat flour, B) powder mixture of raw materials, C) raw dough, and D) baked cookies determined by SE-HPLC using ACN as extraction solution
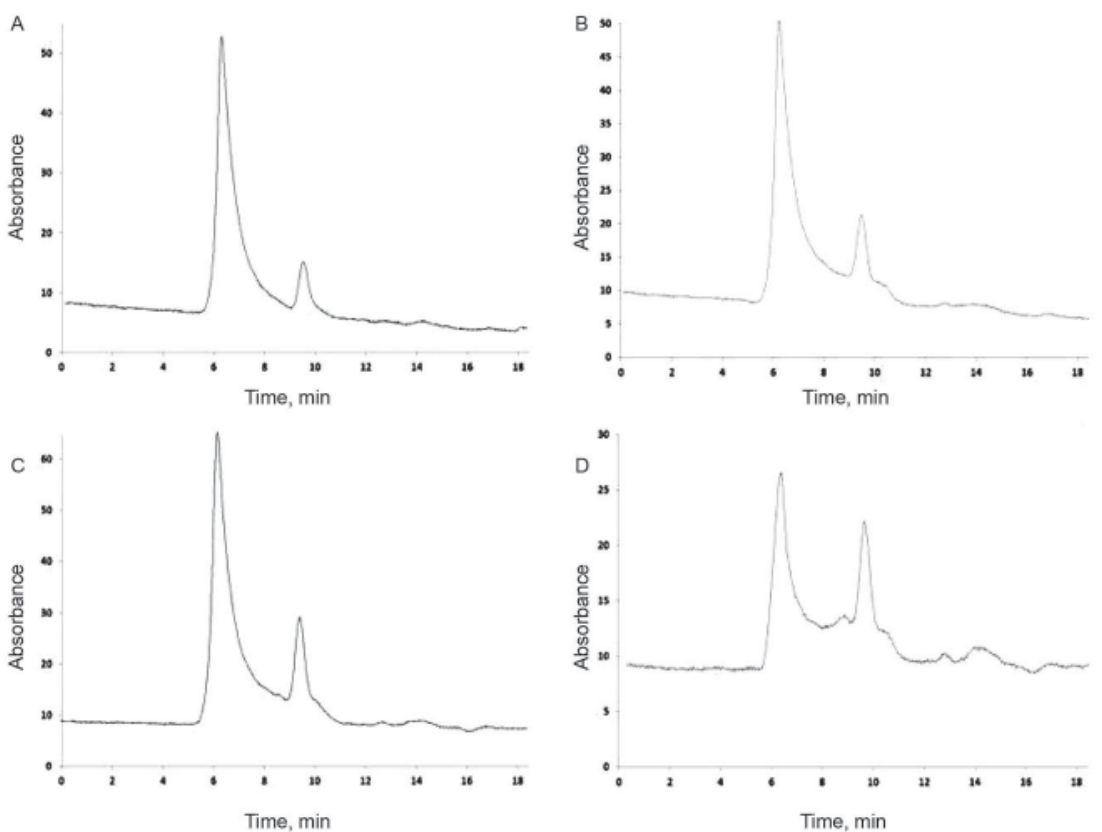

Fig. 4. Insoluble protein fractions of A) wheat flour, B) powder mixture of raw materials, C) raw dough, and D) baked cookies determined by SE-HPLC using ACN as extraction solution 
In case of $70 \%(\mathrm{v} / \mathrm{v})$ ethanol solvent, the amount of low molecular weight proteins is continuously increasing during food processing (Fig. 5). On the contrary, in case of gliadin fractions, the size of peak area is continuously decreasing throughout the production process. The protein profile of insoluble fractions does not change significantly during processing from wheat flour to heat treated cookies (data not shown).
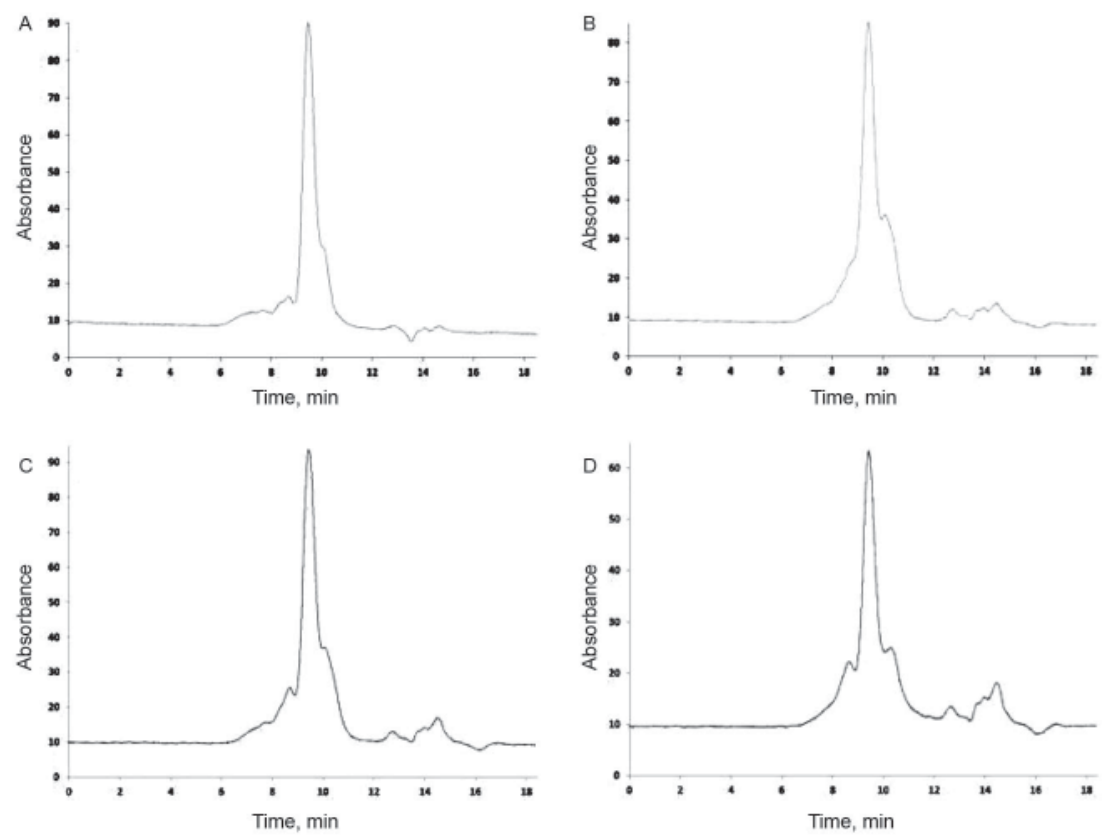

Fig. 5. Soluble fractions of A) wheat flour, B) powder mixture of raw materials, C) raw dough, and D) baked cookies determined by SE-HPLC using ethanol as extraction solution

To sum it up, food processing causes changes in protein profiles and in the size of peak areas in case of both extraction solvents (Table 1). Comparing the results of ELISA and SEHPLC measurements, it could be observed that the trends of changes during processing are similar. Figure 6 shows that the gliadin concentration measured with ELISA method is lower in raw dough than in the mixture of dry materials. But significant decrease could be observed only in baked cookies. The same trend can be observed in case of the SE-HPLC results, as well. In the background, two phenomena can be identified, namely the decrease of soluble protein content after baking and the changes in the ratio of soluble protein fractions, which is affected by the type of the extraction solvent (BorNeO \& KHAN, 1999; SinGH, 2005; LAGRAin et al., 2008). The poorer protein solubility is probably caused by formation of gluten macro polymers with S-S bonds, which could be handled by using a reducing agent in extraction cocktails in ELISA procedures. On the other hand, the rate of changes in measured gliadin concentration in baked cookies is differently measured by different kits. As after the extraction with reducing agent the decrease is still present, it is assumable that other effects might occur, i.e. formation of complexes with other food components and changes in the immunoaffinity of proteins. 
Table 1. Ratio of soluble fractions I to III in the total protein profile (w/w\%). Determination was carried out by SEHPLC. Fraction I: glutenins and $\omega$-gliadins; II: $\alpha$-, $\beta$-, $\gamma$-gliadins; III: low molecular weight proteins

\begin{tabular}{llccc}
\hline Sample & Extraction solution & Fraction I & Fraction II & Fraction III \\
\hline Wheat flour & ACN & 35.40 & 49.11 & 15.48 \\
& $70 \%(\mathrm{v} / \mathrm{v})$ ethanol & 15.60 & 72.03 & 12.37 \\
& ACN & 33.93 & 43.33 & 33.62 \\
Mixture of raw materials & AC\% (v/v) ethanol & 15.00 & 51.38 & 22.60 \\
& ACN & 33.01 & 44.39 & 33.48 \\
Raw dough & $70 \%(\mathrm{v} / \mathrm{v})$ ethanol & 17.12 & 49.40 & 29.41 \\
ACN & $70 \%(\mathrm{v} / \mathrm{v})$ ethanol & 24.70 & 45.90 & 38.97 \\
\hline
\end{tabular}

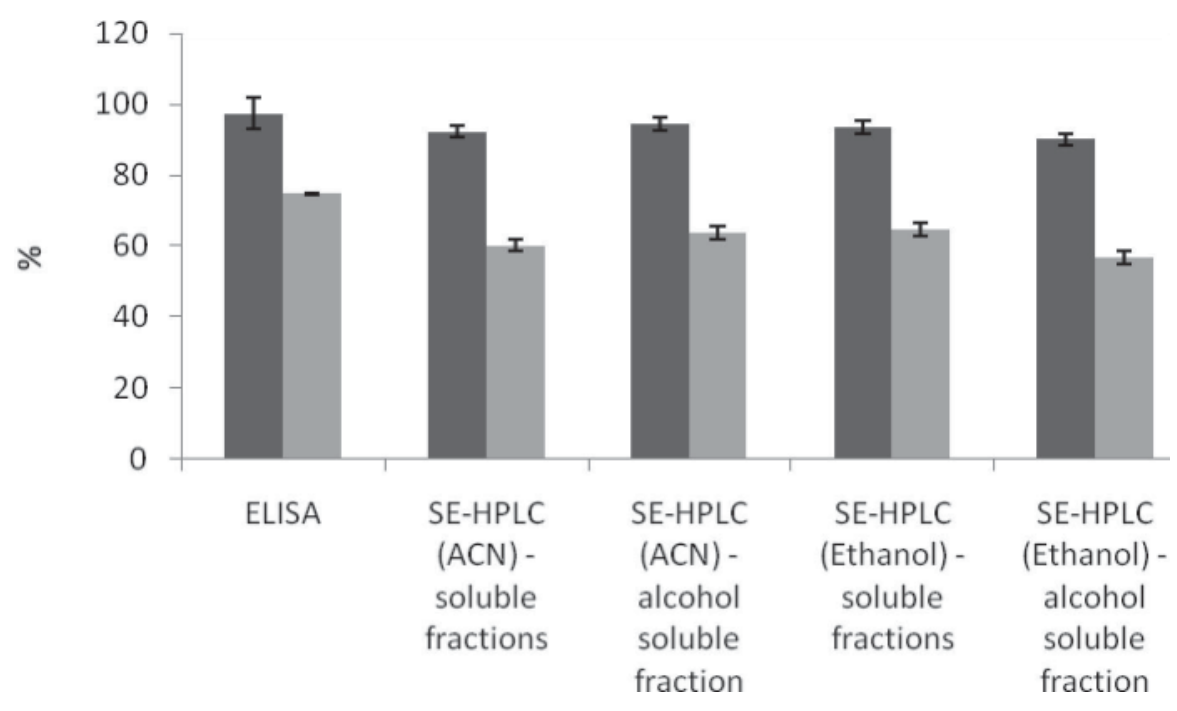

Fig. 6. Changes during food processing measured by ELISA and SE-HPLC. (Bars are representing the extent of decrease of gliadin, soluble fractions and alcohol soluble fractions in the raw dough: $\square$ and in the baked cookies: $\square$ as a percentage of the corresponding data measured in the powder mixture of raw materials.)

\section{Conclusions}

In conclusion it could be observed that the matrix and the production process may have considerable effects on the analytical results. In the two cases described above, it can be seen that both the presence of fat content and the heat treatment cause detectable changes. The decreased measurable gluten concentration described above is in line with the results of 
previously published studies (Borneo \& Khan, 1999; Hayta \& Schoefield, 2004; Singh 2005; KiefFer et al., 2007; Lagrain et al., 2008; GomaA \& Boye, 2013). But without understanding the background of these changes, accurate analytical methods cannot be developed. Using different ELISA methods the extent of the decrease in measurable gluten concentration varies considerably. Consequently, one might conclude that a change in protein extractability during heat treatment is only one factor that affects the analytical measurements. Since ELISA methods are using different extraction protocols and different antibodies as well, the extent of decrease can also be affected by the different changes of different epitopes during heat treatment. In addition, there is no information about how the structure or availability of epitopes changes due to matrix components and how the allergen activity and immune activity changes during processing. Our reference material enables the investigation of the phenomena in a well defined matrix. This advantageous situation helps us to identify the source of analytical errors caused by the mentioned effects and to estimate their order of magnitude. From theoretical and practical points of view, revealing the physical and chemical background of the experienced changes is highly relevant. However, it is important to emphasise that these results are originated from a simplified preliminary study; further investigation is needed to clarify the identified phenomena.

This research is related to the scientific goals of the MoniQA Association and the national project "Development of quality orientated, harmonized educational and R+D $+\mathrm{I}$ strategy and operational model at the Budapest University of Technology and Economics" (ÚMFT TÁMOP-4.2.1/B-09/1/KMR-2010-0002).

\section{References}

A.O.A.C. (2006): Official methods of analysis, Crude fat in feeds, cereal grains and forage. Method 2003.06. AOAC Int., Gaithersburg, MD.

Borneo, R. \& Khan, K. (1999): Protein changes during various stages of breadmaking of four spring wheats: Quantification by size-exclusion HPLC. Cereal Chem., 76, 711-717.

Boye, J.I. \& Godefroy, S.B. (2010): Allergen management in food industry. John Wiley \& Sons Inc., Hoboken, New Jersey, pp. 1-167.

Briani, C., Samaroo, D. \& Alaedini, A. (2008): Celiac disease: From gluten to autoimmunity. Autoimmun. Rev., 7, $644-650$

Bugyi, Z., TÖRÖK, K., Hajas, L., Adonyi, Z., Popping, B. \& TÖMÖSKÖZI, S. (2013): Comparative study of commercially available gluten ELISA kits using an incurred reference material. Qual. Assur. Saf. Crop. (QAS), 5(1), 79-87.

Bugyi, Z., Török, K., Hajas, L., Adonyi, Z., Poms, R., Popping, B., Diaz-Amigo, C., Kerbach, S. \& Tömösközi, S. (2012): Development of incurred reference material for improving conditions of gluten quantification. $J$. AOAC Int., 95, 382-387.

Cabera-Chavez, F. \& Calderon de la Barca, A.M. (2010): Trends in wheat technology and modification of gluten proteins for dietary treatment of celiac disease patients. J. Cereal Sci., 52, 337-341.

GomaA, A. \& Boye, J.I. (2013): Impact of thermal processing time and cookie size on the detection of casein, egg, gluten and soy allergens in food. Food Res. Int., 52, 483-489.

HaYta, M. \& Schofield, J.D. (2004): Heat and additive induced biochemical transitions in gluten from good and poor breadmaking quality wheats. J. Cereal Sci., 40, 245-256.

ISO (1992): ISO Guide 30 Terms and definitions used in connection with reference materials.

KiefFer, R., Schurer, F., KÖHLER, P. \& Wieser, H. (2007): Effect of hydrostatic pressure and temperature on the chemical and functional properties of wheat gluten: Studies on gluten, gliadin and glutenin. J. Cereal Sci., 45, $285-292$.

Koppelman, S.J. \& Hefle, S.L. (2006): Detecting allergens in food. Woodhead Publishing Limited, Cambridge, England, pp. 63-182; 348-356. 
Lagrain, B., Thewissen, B.G., Briss, K. \& Delcour, J.A. (2008): Mechanism of gliadin-glutenin cross-linking during hydrothermal treatment. Food Chem., 107, 753-760.

LARroque, O.R. \& BÉKÉs, F. (2000): Rapid size-exclusion chromatography analysis of molecular size distribution for wheat endosperm protein. Cereal Chem.,77, 451-453.

MANU, B.T. \& RAO, U.J.S.P. (2008): Influence of size distribution of proteins, thiol and disulfide content in wheat flour on rheological and chapati texture of Indian wheat varieties. Food Chem., 110, 88-95.

Rumbo, M., Chirdo, F.G., Fossati, C.A. \& Anon, M.C. (2001): Analysis of the effects of heat treatment on gliadin immunochemical quantification using a panel of anti-prolamin antibodies. J. Agric. Food Chem., 49, 57195726.

Sathe, S.K., Teuber, S.S. \& Roux, K.H. (2005): Effects of food processing on the stability of food allergens. Biotechnol. Adv., 23, 423-429.

Singh, H. \& MacRitchie, F. (2004): Changes in proteins induced by heating gluten dispersions at high temperature. J. Cereal Sci., 39, 297-301.

SINGH, H. (2005): A study of changes in wheat protein during bread baking using SE-HPLC. Food Chem., 90, 247250.

Taylor, S.L. \& Hefle, S.L. (2001): Food allergies and other food sensitivities. Food Technol., 55(9), 470-475. 